\title{
New year, new science
}

\section{Nature looks ahead to the key findings and events that may emerge in 2013.}

\section{STEM-CELL TRIALS}

Landmark results from an early-stage clinical trial using human embryonic stem cells (hESCs) should appear this year. Biotechnology firm Advanced Cell Technology of Santa Monica, California, is injecting hESC-derived retinal cells into the eyes of around three dozen people with two forms of non-treatable degenerative blindness. It is the only company currently testing hESC therapies with US Food and Drug Administration (FDA) approval, and it hopes that the agency will give it the green light to test stem cells induced from adult cells in patients this year.

\section{DIAGNOSTICS CONTROVERSY}

The American Psychiatric Association will publish the fifth edition of its Diagnostic and Statistical Manual of Mental Disorders (DSM-5) in May, the first major update in 19 years to the standard reference guide for diagnosing mental illnesses. It will lead to controversial changes in clinical and research protocols, including restructured diagnoses for autism and major depression, although as a 'living document' the DSM-5 will see further revisions.

\section{CLIMATE ASSESSMENT}

Climate scientists have spent years preparing the fifth assessment report from the Intergovernmental Panel on Climate Change, its first update since 2007. Part of that report is due to appear in September: the conclusions of Working Group I, which summarizes the basic science of global warming. In the United States, the Global Change Research Program's second assessment will detail the national impacts of climate change.

\section{THE BIG BANG'S GLOW}

One of the stunning images of the year could be provided by the comet ISON, which will pass close to the Sun in November and could outshine the full Moon as its surface boils away into space. Just as spectacular will be the Planck space telescope's map of the faint microwave afterglow from the Big Bang, which could even reveal ripples from gravitational waves generated during an initial period of cosmic 'inflation'. In other missions, NASA's LADEE spacecraft will orbit the Moon to study lunar dust; its MAVEN mission will launch to explore Mars' upper atmosphere; and the Curiosity rover will

\section{DNATURE.COM} For a look back at the highlights of 2012, see: www.nature.com/2012 continue to send back results from the red planet's surface. Back on Earth, the massive 66-dish Atacama Large Millimeter/submillimeter Array in Chile will be completed.

\section{DIET, MICROBES AND CANCER}

Scientists increasingly suspect that our intestinal zoo of microbes might be the key link between diet and diseases such as cancer. A study last year connected a higher-than-normal proportion of the bacterium Escherichia coli to colorectal cancer in mice with inflam-

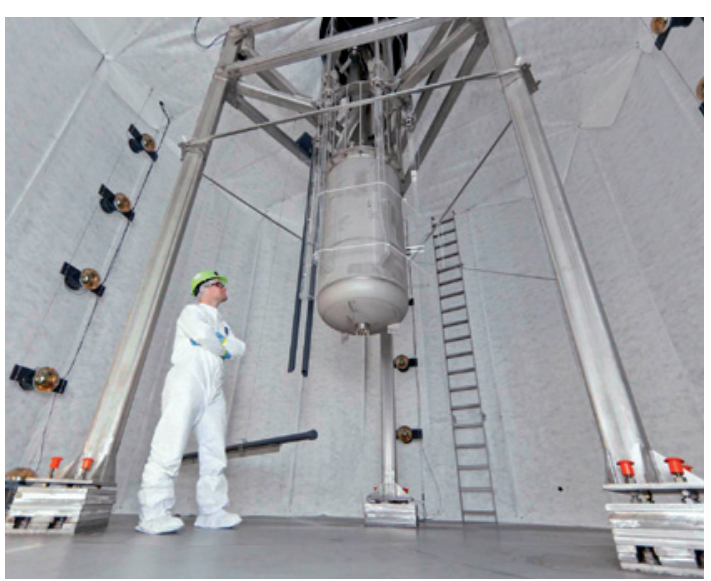

The Large Underground Xenon detector is seeking dark matter.

matory bowel disease (J. C. Arthur et al. Science 338, 120-123; 2012). More studies this year will unpick the effect of diets on the gut microbiome and their implications for disease risk. Meanwhile, GlaxoSmithKline should find out whether the FDA approves its melanoma treatment trametinib, potentially the first in a new class of compounds that inhibit a kinase signalling pathway regulating cell growth.

\section{PARTICLE SEEKING}

After contradictory sightings of dark-matter particles from various underground experiments, the Large Underground Xenon detector at the Sanford Underground Research Facility in Lead, South Dakota, may this year boost or rule out some of the claims. The king of particle hunters - the Large Hadron Collider at CERN near Geneva - will shut down until 2015 for an upgrade to enable more powerful collisions, but physicists will continue to pore over the data collected so far for hints of supersymmetry.

\section{THE LOWER DEPTHS}

Data will start flowing from the first completed segments of a giant underwater surveillance network, the US Ocean Observatories Initiative, which will cost US\$386 million to build and will be completed by March 2015. It will monitor everything from undersea earthquakes and the effects of climate change on ocean circulation, to shifting ecosystems and ocean chemistry - all the way from the air to the seabed at seven sites around the globe. Meanwhile, British, American and Russian teams will be hoping to find out what kind of life, if any, exists in three deep, subglacial Antarctic lakes.

\section{MAGICAL MATERIALS}

Samarium hexaboride might be the next star of materials science, following hints last year that it is a topological insulator - conducting electricity on its surface, but behaving as an insulator inside. Graphene will remain a major celebrity, so expect a flood of reports about copycat materials such as boron nitride, tantalum disulphide and other two-dimensional sheets that can be stacked or sandwiched in precise layers.

\section{GENES IN COURT}

The US Supreme Court could decide a number of cases with science implications in 2013. It will re-examine whether genes are patentable as part of a threeyear lawsuit considering the validity of patents held by Myriad Genetics in Salt Lake City, Utah. It may also rule on a challenge to seed firm Monsanto, based in St Louis, Missouri, from a farmer who wants to plant seeds gathered from previously grown genetically modified crops, rather than buying new stock from the company. And the court will consider whether brand-name pharmaceutical companies can pay generic makers to delay their launch of generic drugs.

\section{PAPER MONEY}

A UK policy that requires publicly funded researchers to make their results freely available will take effect from April. Other countries could soon follow - a Global Research Council meeting is set to discuss the matter in May. But many scientists will be worrying more about budgets, with the United States considering drastic spending cuts that could take effect early this year (see page 13), and Europe set to continue debating the proposed $€ 80$ billion (US\$104 billion) in funding for its 2014-20 research programme, Horizon 2020.

COMPILED BY RICHARD VAN NOORDEN 